\title{
Mass differences of light hadron isomultiplets
}

\author{
B. E. Palladino and P. Leal Ferreira \\ Instituto de Física Téorica, Universidade Estadual Paulista, Rua Pamplona 145, 01405 São Paulo, São Paulo, Brazil
}

(Received 12 June 1989)

\begin{abstract}
Mass differences of low-lying, nonstrange, hadron isomultiplets are investigated in the framework of a relativistic, independent quark potential model, implemented by center-of-mass, one-gluonexchange, and pion-cloud corrections. The introduction of pionic self-energy corrections with nondegenerate intermediate states is instrumental in our analysis, playing also a fundamental role for a successful description of the $\rho-\omega$ mass splitting. The effect of the superposition of all these corrections is discussed in some detail for the $p-n, \pi^{+}-\pi^{0}, \rho^{+}-\rho^{0}$, and $\Delta^{++}-\Delta^{0}$ mass differences. The corresponding hadronic masses are also calculated with suitable values for the hadronic sizes and quark masses.
\end{abstract}

\section{INTRODUCTION}

The problem of hadronic isomultiplet mass differences (IMMD's) has been a subject of continuous interest in the existing literature. Although isospin mass differences are often called electromagnetic mass differences, it is well known that the electromagnetic interaction is not the only source of the effect since quark mass differences such as $m_{d}-m_{u}$ induce appreciable contributions from strong interactions, ${ }^{1}$ which stem from one-gluon-exchange (OGE) and pion-cloud corrections.

In the present paper, IMMD's of light nonstrange hadrons are investigated in the framework of a relativistic, independent, $S+V$ harmonic quark potential model, ${ }^{2}$ which has been implemented by one-gluon-exchange, center-of-mass, ${ }^{3}$ and pion-cloud corrections. ${ }^{4}$ Pion-cloud corrections were introduced in the model in order to incorporate chiral symmetry, which is a fundamental property of QCD associated to its massless $(u, d) \mathrm{SU}(2)$-flavor sector. $^{5}$ In the context of the $S+V$ model, contrarily to the quark vector current, the quark axial-vector current is not conserved due to the presence of the Lorentz-scalar component $S$ of the confining potential. In order to restore chiral symmetry, an elementary Goldstone pion field is introduced interacting with the quarks of the bare "core" in a linearized way. ${ }^{4}$ As a consequence, pionexchange and self-energy effects give rise to additional contributions to the hadronic mass spectrum. In the same spirit of the cloudy-bag model, ${ }^{6}$ these pionic effects are assumed to be small (or moderate) and are treated perturbatively in lowest order of the quark-pion constant.

The present treatment of the IMMD's of nonstrange low-lying hadrons, based on the above-defined model, incorporates besides specific one-photon-exchange contributions, the contributions of one-gluon-exchange and pion-cloud corrections resulting from the $m_{d}-m_{u}$ mass difference. We note that a similar approach for studying the mass differences among $\mathrm{SU}(3)$ baryon multiplets has also been undertaken by Hwang, ${ }^{7}$ based on the MIT bag model, ${ }^{8}$ with part of the flavor-SU(3) violations arising out of pion-cloud effects. A high-quality fit of the baryon masses was obtained by means of a best fit of the chiral- bag parameters, corresponding to a nucleon bag radius of around $0.45 \mathrm{fm}$. However, the mesonic case was not treated in that work. On the other hand, Itoh et al., ${ }^{9}$ based on a semirelativistic quark potential model with Gaussian wave functions, have discussed in detail the IMMD's for both low-lying baryons and mesons. They introduced explicitly a flavor-SU(6)-symmetry breaking in the Gaussian wave functions for describing the baryons, in order to take into account spin-spin effects stemming from OGE, an effect which is also instrumental to explain the negative charge radius of the neutron. As we shall discuss here, similarly to Ref. 9, the mesonic sector requires an appropriate parametrization different from that used for baryons. Furthermore, a better understanding of the effects involved in the IMMD's, both for baryons and mesons, seems to require the consideration of the available intermediate states associated to the Goldstone pion, in lowest order. Thus, in our treatment of the pion cloud a novel feature is that the Goldstone pion self-energy corrections are calculated including the contributions of the different intermediate states $\left(0^{-}\right.$and $1^{-}$for mesons, spin $\frac{1}{2}$ and $\frac{3}{2}$ for baryons), either in the case in which they are degenerate in mass or in the case this mass degeneracy is broken by OGE and electromagnetic corrections. As we shall see, a correct estimate of the hadronic size (described by its bag radius or, in the present model, by the Gaussian radius of the constituent quarks) will play a fundamental role in our analysis.

The paper is organized as follows. In Sec. II, we briefly review the $S+V$ potential model and discuss the necessary expressions for calculating the hadronic IMMD's. Section III is devoted to the exposition of the results obtained and Sec. IV to our final remarks and conclusions.

\section{BASIC FRAMEWORK}

We begin this section by briefly recalling our singlequark, $S$-wave framework. In the $S+V$ potential model each quark in the hadron obeys a Dirac equation ${ }^{2}$

$$
\left[\boldsymbol{\alpha} \cdot \mathbf{p}+\beta m_{i}+\frac{1}{2}(1+\beta) V(r)\right] \psi_{i}(\mathbf{r})=E_{i} \psi_{i}(\mathbf{r}),
$$

where $i$ is the quark flavor index. For reasons of simplici- 
ty, we have adopted for the confining potential $V(r)$ the harmonic form

$$
V(r)=V_{0}+\frac{1}{2} K r^{2} .
$$

This choice allows a simpler, analytical treatment and gives results very close to those obtained with the linear potential $^{2}$ both for the hadronic mass spectrum and static properties. The $S$-wave solution of Eq. (1) is

$$
\psi_{i}(r)=N_{i}\left[\begin{array}{c}
\phi_{i}(r) \chi \\
\frac{1}{x_{i}} \sigma \cdot \mathbf{p} \phi_{i}(r) \chi
\end{array}\right]
$$

where $x_{i}=E_{i}+m_{i}$, the normalization constant is given by $N_{i}^{2}=\left[1+\frac{3}{2}\left(x_{i} R_{i}\right)^{-2}\right]^{-1}, \chi$ is a Pauli spinor and $\phi(r)$ is a normalized eigenfunction of the radial equation $\mathbf{p}^{2} \phi_{i}(r)=x_{i}\left[E_{i}-m_{i}-V(r)\right] \phi_{i}(r)$, which has the form

$$
\phi_{i}(r)=\left(\frac{1}{\pi R_{i}^{2}}\right)^{3 / 4} e^{-r^{2} / 2 R_{i}^{2}}
$$

with the "Gaussian radius" of the $i$ th constituent quark defined by

$$
R_{i}=\left(\frac{2}{x_{i} K}\right)^{1 / 4}
$$

For the $S$-wave single-quark energy we have

$$
E_{i}=m_{i}+V_{0}+\frac{3}{2} K R_{i}^{2} \text {. }
$$

We note that the spin dependence of the quark confinement potential is, in principle, not known. Among the mixed potentials of the form $a S+b V$, the choice of an equally mixed term $\frac{1}{2}(1+\beta) V(r)$ in Eq. (1) of the present $S+V$ model is motivated by reasons of simplicity and represents, in a phenomenological way, the nonperturbative multigluon interactions which give rise to quark confinement according to QCD.

The hadron masses $M$ can be written in the form

$$
M=\left[\left(E_{0}+E_{g}+E_{\gamma}\right)^{2}-\left\langle P^{2}\right\rangle\right]^{1 / 2}+\Delta E_{\pi},
$$

where $E_{0}=\sum_{i} E_{i}$ is the sum of the single-particle energies, $E_{g}=\Delta E_{M}^{g}+\Delta E_{E}^{g}$ is the sum of the magnetic and electric parts of the one-gluon exchange, ${ }^{2,3} E_{\gamma}$ $=\Delta E_{M}^{\gamma}+\Delta E_{E}^{\gamma}$ is the one-photon-exchange correction, $\left\langle P^{2}\right\rangle^{1 / 2}$ is the center-of-mass energy correction ${ }^{3}$ and $\Delta E_{\pi}$ is the one-pion-exchange self-energy contribution. ${ }^{4}$ These several energy-correction terms are given by

$$
\begin{aligned}
& \Delta E_{M}^{g}=\alpha_{s}\left\langle H\left|\sum_{i, j}\left(\sum_{a} \lambda_{i}^{a} \lambda_{j}^{a}\right)\left(\sigma_{i} \cdot \sigma_{j}\right) I_{i j}^{M}\right| H\right\rangle \\
& =\alpha_{s} \sum_{i, j} a_{i j} I_{i j}^{g M} \text {, } \\
& \Delta E_{E}^{g}=\alpha_{s}\left\langle H\left|\sum_{i, j}\left(\sum_{a} \lambda_{i}^{a} \lambda_{j}^{a}\right) I_{i j}^{E}\right| H\right\rangle=\alpha_{s} \sum_{i, j} b_{i j} I_{i j}^{g E}, \\
& \Delta E_{M}=\alpha\left\langle H\left|\sum_{i, j}\left(Q_{i} Q_{j}\right)\left(\sigma_{i} \cdot \sigma_{j}\right) I_{i j}^{M}\right| H\right\rangle=\alpha \sum_{i, j} a_{i j}^{\prime} I_{i j}^{\gamma M}, \\
& \Delta E_{E}^{\gamma}=\alpha\left\langle H\left|\sum_{i, j}\left(Q_{i} Q_{j}\right) I_{i j}^{E}\right| H\right\rangle=\alpha \sum_{i, j} b_{i j}^{\prime} I_{i j}^{\gamma E}
\end{aligned}
$$

where $\alpha_{s}=g_{q g}^{2} / 4 \pi$ is the strong-coupling constant, $\alpha=e^{2} / 4 \pi=\frac{1}{137}$ is the fine-structure constant, $\lambda_{i}^{a}$ are the Gell-Mann matrices of the SU(3)-color group, $\sigma_{i}$ represents the Pauli spin and $Q_{i}$ is the charge of the $i$ th quark. In Eqs. (8) and (9) we are using the definitions $I_{i j}^{\gamma} \equiv-\frac{3}{16} I_{i j}^{g} \equiv I_{i j}$, with

$$
\begin{aligned}
I_{i j}^{M}=-\frac{4}{3 \sqrt{\pi}} \frac{1}{\left(R_{i}^{2}+R_{j}^{2}\right)^{3 / 2}} \frac{1}{x_{i} x_{j}} N_{i}^{2} N_{j}^{2}, \\
\begin{aligned}
\left.I_{i j}^{E}=\frac{1}{\sqrt{\pi}} \frac{1}{\left(R_{i}^{2}+R_{j}^{2}\right)^{3 / 2}}\left[x_{i} x_{j}\left(R_{i}^{2}+R_{j}^{2}\right)+\frac{x_{i}}{x_{j}}\left[1+\frac{3}{2} \frac{R_{i}^{2}}{R_{j}^{2}}\right]+\frac{x_{j}}{x_{i}}\right] 1+\frac{3}{2} \frac{R_{j}^{2}}{R_{i}^{2}}\right] \\
\left.+\frac{15}{4} \frac{1}{x_{i} x_{j}\left(R_{i}^{2}+R_{j}^{2}\right)}\left[1+\frac{2}{5} \frac{R_{i}^{4}+R_{j}^{4}}{R_{i}^{2} R_{j}^{2}}\right]\right] \frac{1}{x_{i} x_{j}} N_{i}^{2} N_{j}^{2} .
\end{aligned}
\end{aligned}
$$

Details of this calculation can be found in Ref. 3 . Analogously to Eqs. (8) and (9) one can also obtain ${ }^{4}$

$$
\Delta E_{\pi}=\alpha_{\pi}\left\langle H\left|\sum_{i, j}\left(\tau_{i} \cdot \tau_{j}\right)\left(\sigma_{i} \cdot \sigma_{j}\right) I_{i j}^{\pi}\right| H\right\rangle=\alpha_{\pi} \sum_{i, j} c_{i j} I_{i j}^{\pi}
$$

where $\alpha_{\pi}=g_{q \pi}^{2} / 4 \pi$ is an effective quark-pion coupling constant and the $\tau_{i}$ are expressed in terms of Pauli isospin matrices associated to the $i$ th quark.

The coefficients $a_{i j}, b_{i j}$, and $c_{i j}$ which appear in Eqs. (8), (9), and (11) can be easily evaluated and are given in Table I, for the hadrons considered in this work. Al- though we have performed our calculations with the inclusion of the self-energy terms ${ }^{10}$ [terms with $i=j$ in Eqs. (8), (9), and (11)], which give rise to the coefficients in Table $I(a)$, we also display in Table $I(b)$ the coefficients obtained without the inclusion of the self-energies, as a possible useful reference.

Now, we would like to concentrate our discussion in the pionic energy corrections. An alternative expression to that of Eq. (11) is ${ }^{4}$

$$
\Delta E_{\pi}=-\frac{1}{3} f_{N N \pi}^{2} \frac{9}{25} C(H) I_{\pi},
$$


TABLE I. Coefficients $a_{i j}, b_{i j}$, and $c_{i j}$, defined in the text by Eqs. (8), (9), and (11), evaluated for the low-lying light hadrons, including self-energy terms (a) and without self-energy terms (b).

\begin{tabular}{|c|c|c|c|c|c|c|c|c|c|c|c|c|}
\hline \multicolumn{5}{|c|}{ One-gluon exchange } & \multicolumn{6}{|c|}{ One-photon exchange } & \multirow{2}{*}{\multicolumn{2}{|c|}{$\begin{array}{l}\text { One-pion } \\
\text { exchange }\end{array}$}} \\
\hline Magneti & $\operatorname{art}$ & & lectri & part & & gnet & part & & ectri & part & & \\
\hline$a_{u d}$ & $a_{d d}$ & $b_{u u}$ & $b_{u d}$ & $b_{d d}$ & $a_{u u}^{\prime}$ & $a_{u d}^{\prime}$ & $a_{d d}^{\prime}$ & $b_{u u}^{\prime}$ & $b_{u d}^{\prime}$ & $b_{d d}^{\prime}$ & $c_{u u}$ & $c_{u d}$ \\
\hline
\end{tabular}

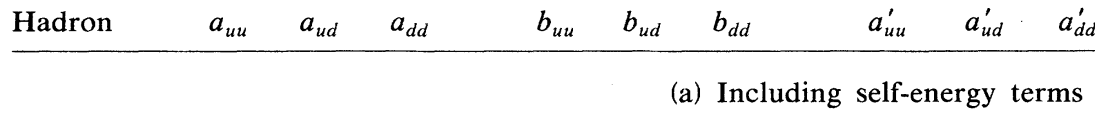

\begin{tabular}{|c|c|c|c|c|c|c|c|c|c|c|c|c|c|c|c|}
\hline \\
\hline$P$ & -5 & -4 & -3 & -1 & 2 & -1 & $-\frac{16}{9}$ & $\frac{16}{9}$ & $-\frac{1}{3}$ & 0 & $-\frac{8}{9}$ & $-\frac{1}{9}$ & 20 & 28 & 9 \\
\hline$N$ & -3 & -4 & -5 & -1 & 2 & -1 & $-\frac{4}{3}$ & $\frac{16}{9}$ & $-\frac{4}{9}$ & $-\frac{4}{9}$ & $-\frac{8}{9}$ & 0 & 9 & 28 & 20 \\
\hline$\Delta^{++}$ & -6 & 0 & 0 & 0 & 0 & 0 & $-\frac{4}{3}$ & 0 & 0 & $\frac{4}{3}$ & 0 & 0 & 33 & 0 & 0 \\
\hline$\Delta^{+}$ & -5 & 2 & -3 & -1 & 2 & -1 & $-\frac{16}{9}$ & $-\frac{8}{9}$ & $-\frac{1}{3}$ & 0 & $-\frac{8}{9}$ & $-\frac{1}{9}$ & 20 & 4 & 9 \\
\hline$\Delta^{0}$ & -3 & 2 & -5 & -1 & 2 & -1 & $-\frac{4}{3}$ & $-\frac{8}{9}$ & $-\frac{4}{9}$ & $-\frac{4}{9}$ & $-\frac{8}{9}$ & 0 & 9 & 4 & 20 \\
\hline$\Delta^{-}$ & 0 & 0 & -6 & 0 & 0 & 0 & 0 & 0 & $-\frac{1}{3}$ & 0 & 0 & $\frac{1}{3}$ & 0 & 0 & 33 \\
\hline$\rho^{+}$ & -3 & 2 & -3 & -1 & 2 & -1 & $+\frac{4}{3}$ & $+\frac{4}{9}$ & $+\frac{1}{3}$ & $+\frac{4}{9}$ & $+\frac{4}{9}$ & $+\frac{1}{9}$ & 9 & -2 & 9 \\
\hline$\rho^{0}$ & -2 & 0 & -2 & 0 & 0 & 0 & $+\frac{8}{9}$ & 0 & $+\frac{2}{9}$ & 0 & 0 & 0 & 8 & 0 & 8 \\
\hline$\rho^{-}$ & -3 & 2 & -3 & -1 & 2 & -1 & $+\frac{4}{3}$ & $+\frac{4}{9}$ & $+\frac{1}{3}$ & $+\frac{4}{9}$ & $+\frac{4}{9}$ & $+\frac{1}{9}$ & 9 & -2 & 9 \\
\hline$\omega^{0}$ & -2 & 0 & -2 & 0 & 0 & 0 & $+\frac{8}{9}$ & 0 & $+\frac{2}{9}$ & 0 & 0 & 0 & 12 & 0 & 12 \\
\hline$\pi^{+}$ & -3 & -6 & -3 & -1 & 2 & -1 & $+\frac{4}{3}$ & $-\frac{4}{3}$ & $+\frac{1}{3}$ & $+\frac{4}{9}$ & $+\frac{4}{9}$ & $+\frac{1}{9}$ & 9 & 6 & 9 \\
\hline$\pi^{0}$ & -6 & 0 & -6 & 0 & 0 & 0 & $+\frac{8}{3}$ & 0 & $+\frac{2}{3}$ & 0 & 0 & 0 & 12 & 0 & 12 \\
\hline$\pi^{-}$ & -3 & -6 & -3 & -1 & 2 & -1 & $+\frac{4}{3}$ & $-\frac{4}{3}$ & $+\frac{1}{3}$ & $+\frac{4}{9}$ & $+\frac{4}{9}$ & $+\frac{1}{9}$ & 9 & 6 & 9 \\
\hline
\end{tabular}

(b) Without self-energy terms

\begin{tabular}{cccccccccccccccc}
$P$ & 1 & -4 & 0 & 1 & 2 & 0 & $\frac{8}{9}$ & $\frac{16}{9}$ & 0 & $\frac{8}{8}$ & $-\frac{8}{9}$ & 0 & 2 & 28 & 0 \\
$N$ & 0 & -4 & 1 & 0 & 2 & 1 & 0 & $\frac{16}{9}$ & $\frac{2}{9}$ & 0 & $-\frac{8}{9}$ & $\frac{2}{9}$ & 0 & 28 & 2 \\
$\Delta^{++}$ & 3 & 0 & 0 & 3 & 0 & 0 & $\frac{8}{3}$ & 0 & 0 & $\frac{8}{3}$ & 0 & 0 & 6 & 0 & 0 \\
$\Delta^{+}$ & 1 & 2 & 0 & 1 & 2 & 0 & $\frac{8}{9}$ & $-\frac{8}{9}$ & 0 & $\frac{8}{9}$ & $-\frac{8}{9}$ & 0 & 2 & 4 & 0 \\
$\Delta^{0}$ & 0 & 2 & 1 & 0 & 2 & 1 & 0 & $-\frac{8}{9}$ & $\frac{2}{9}$ & 0 & $-\frac{8}{9}$ & $\frac{2}{9}$ & 0 & 4 & 2 \\
$\Delta^{-}$ & 0 & 0 & 3 & 0 & 0 & 3 & 0 & 0 & $\frac{2}{3}$ & 0 & 0 & $\frac{2}{3}$ & 0 & 0 & 6 \\
$\rho^{+}$ & 0 & 2 & 0 & 0 & 2 & 0 & 0 & $+\frac{4}{9}$ & 0 & 0 & $+\frac{4}{9}$ & 0 & 0 & -2 & 0 \\
$\rho^{0}$ & 1 & 0 & 1 & 1 & 0 & 1 & $-\frac{4}{9}$ & 0 & $-\frac{1}{9}$ & $-\frac{4}{9}$ & 0 & $-\frac{1}{9}$ & -1 & 0 & -1 \\
$\rho^{-}$ & 0 & 2 & 0 & 0 & 2 & 0 & 0 & $+\frac{4}{9}$ & 0 & 0 & $+\frac{4}{9}$ & 0 & 0 & -2 & 0 \\
$\omega^{0}$ & 1 & 0 & 1 & 1 & 0 & 1 & $-\frac{4}{9}$ & 0 & $-\frac{1}{9}$ & $-\frac{4}{9}$ & 0 & $-\frac{1}{9}$ & 3 & 0 & 3 \\
$\pi^{+}$ & 0 & -6 & 0 & 0 & 2 & 0 & 0 & $-\frac{4}{3}$ & 0 & 0 & $+\frac{4}{9}$ & 0 & 0 & 6 & 0 \\
$\pi^{0}$ & -3 & 0 & -3 & 1 & 0 & 1 & $+\frac{4}{3}$ & 0 & $+\frac{1}{3}$ & $-\frac{4}{9}$ & 0 & $-\frac{1}{9}$ & 3 & 0 & 3 \\
$\pi^{-}$ & 0 & -6 & 0 & 0 & 2 & 0 & 0 & $-\frac{4}{3}$ & 0 & 0 & $+\frac{4}{9}$ & 0 & 0 & 6 & 0 \\
\hline \hline
\end{tabular}

where, by use of the familiar Goldberger-Treiman relation, we have introduced a parametrization for the model by means of the pseudovector nucleon-pion coupling constant $f_{N N \pi}\left(f_{N N \pi}^{2}=0.08\right)$. In Eq. (12), $C(H)$ is the hadron spin-isospin matrix element, given by

$$
C(H)=\sum_{i, j} c_{i j}=\left\langle H\left|\sum_{i, j} \sigma_{i} \cdot \sigma_{j} \tau_{i} \cdot \tau_{j}\right| H\right\rangle
$$

and

$$
I_{\pi}=\frac{1}{\pi m_{\pi}^{2}} \int_{0}^{\infty} \frac{k^{4}}{\omega_{\pi}^{2}} u^{2}(k) d k, \quad \omega_{\pi}^{2}=k^{2}+m_{\pi}^{2}
$$

where $k$ is the momentum of the interchanged pion and

$$
u(k)=\left(1-A R_{0}^{2} k^{2}\right) e^{-k^{2} R_{0}^{2} / 4}
$$

with

$$
A=\frac{E_{0}-m_{0}}{2\left(5 E_{0}+7 m_{0}\right)}
$$

is the form-factor characteristic of the harmonic $S+V$ model. In the above and subsequent equations, the subscript zero indicates ordinary ( $u$ or $d$ quarks).

We want to note that the presence of a form factor in Eq. (14) is fundamental to obtain the convergence of the integrals $I_{\pi}$. Besides, all the model dependence will be contained in $u(k)$. We also note that the expression for the pion-correction $\Delta E_{\pi}$, Eq. (12), can also be applied to the cloudy bag model ${ }^{6}(\mathrm{CBM})$, provided we take for the form factor the well-known expression $u(k)=3 j_{1}\left(k R_{b}\right)$ / $k R_{b}$, where $R_{b}$ is the bag radius. 


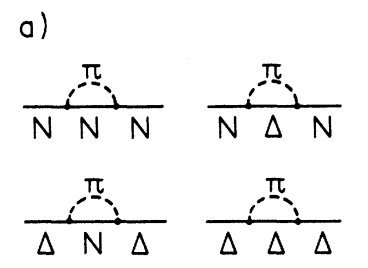

b)
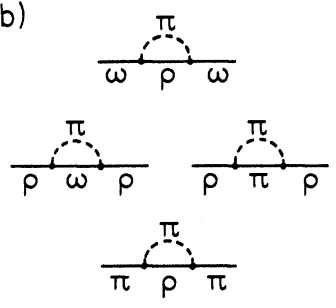

FIG. 1. Available self-energy diagrams for (a) $N$ and $\Delta$ baryons and (b) $\omega, \rho$, and $\pi$ mesons.

The pionic self-energy corrections, as given by Eq. (12), although they consider just one-pion exchange, have been applied successfully in the description of the mass spectrum of low-lying $S$-wave baryons. ${ }^{4}$ However, to attack the mesonic sector an improvement is necessary: Eq. (12) has to include the possibility of contributions of other hadronic intermediate states $H^{\prime}$ belonging to the same multiplet of the hadron $H$. These contributions will depend on the mass differences $M_{H^{\prime}}-M_{H}$, which are nonvanishing for $H^{\prime} \neq H$ because we assume that the mass degeneracy has already been broken due to color OGE (Ref. 11). For degenerate intermediate-energy states, the spinisospin matrix element $C(H)$ in Eq. (9) can be decomposed in a sum over all the available intermediate states $H^{\prime}$ : namely,

$C(H)=\sum_{H^{\prime}} C\left(H H^{\prime}\right)=\sum_{H^{\prime}} \sum_{i, j}\left\langle H\left|\sigma_{i} \tau_{i}\right| H^{\prime}\right\rangle\left\langle H^{\prime}\left|\sigma_{j} \tau_{j}\right| H\right\rangle$,

where each term $C\left(H H^{\prime}\right)$ is the matrix transition element associated to the vertex $H H^{\prime} \pi$ of the corresponding oneGoldstone-pion-exchange diagram. The available diagrams to the hadrons considered in the present work are shown in Fig. 1. When states $H$ and $H^{\prime}$ no longer degenerate are introduced, ${ }^{11}$ we shall have

$$
C^{\prime}(H)=\sum_{H^{\prime}} \delta_{H}\left(H^{\prime}\right) C\left(H H^{\prime}\right),
$$

where

$$
\delta_{H}\left(H^{\prime}\right)=\frac{\int_{0}^{\infty} \frac{k^{4} u^{2}(k) d k}{\omega_{\pi}\left(\omega_{\pi}+M_{H^{\prime}}-M_{H}\right)}}{\int_{0}^{\infty} \frac{k^{4} u^{2}(k)}{\omega_{\pi}^{2}} d k},
$$

with $\delta_{H}(H)=1$.

The integrals in $\delta_{H}\left(H^{\prime}\right)$ are performed numerically. Firstly, it is convenient to make the change of variables $x=k^{2} / m_{\pi}^{2} \Longrightarrow \omega_{\pi}=m_{\pi}(x+1)^{1 / 2}$, leading to

$\delta_{H}\left(H^{\prime}\right)=\frac{m_{\pi}}{2 \pi I_{\pi}} \int_{0}^{\infty} \frac{x^{3 / 2} u^{2}(x) d x}{(x+1)\left[1+d(x+1)^{-1 / 2}\right]}$,

where we have defined

$$
d=\frac{\delta_{M}}{m_{\pi}}=\frac{M_{H^{\prime}}-M_{H}}{m_{\pi}} .
$$

In Eq. (18), the form factors for the $S+V$ and CB model can be written, respectively, as

$$
u^{2}(x)=(1-2 A z x)^{2} e^{-z x},
$$

where $z=\frac{1}{2} m_{\pi}^{2} R_{0}^{2}$ and

$u^{2}(x)=\frac{9}{\left(2 z^{\prime} x\right)^{3}}\left[\sin \left(2 z^{\prime} x\right)^{1 / 2}-\left(2 z^{\prime} x\right)^{1 / 2} \cos \left(2 z^{\prime} x\right)^{1 / 2}\right]^{2}$,

where $z^{\prime}=\frac{1}{2} m_{\pi}^{2} R_{b}^{2}$.

We evaluated the intermediate-mass corrections $\delta_{H}\left(H^{\prime}\right)$ given by Eqs. (17) and (18) as a function of the mass difference $\delta_{M}=M_{H^{\prime}}-M_{H}$ for several values of the parameter $R_{0}$, defined by Eq. (5), and plotted the curves given in Fig. 2. We also calculated these corrections for the CBM for a bag radius of $1 \mathrm{fm}$, using the form factor

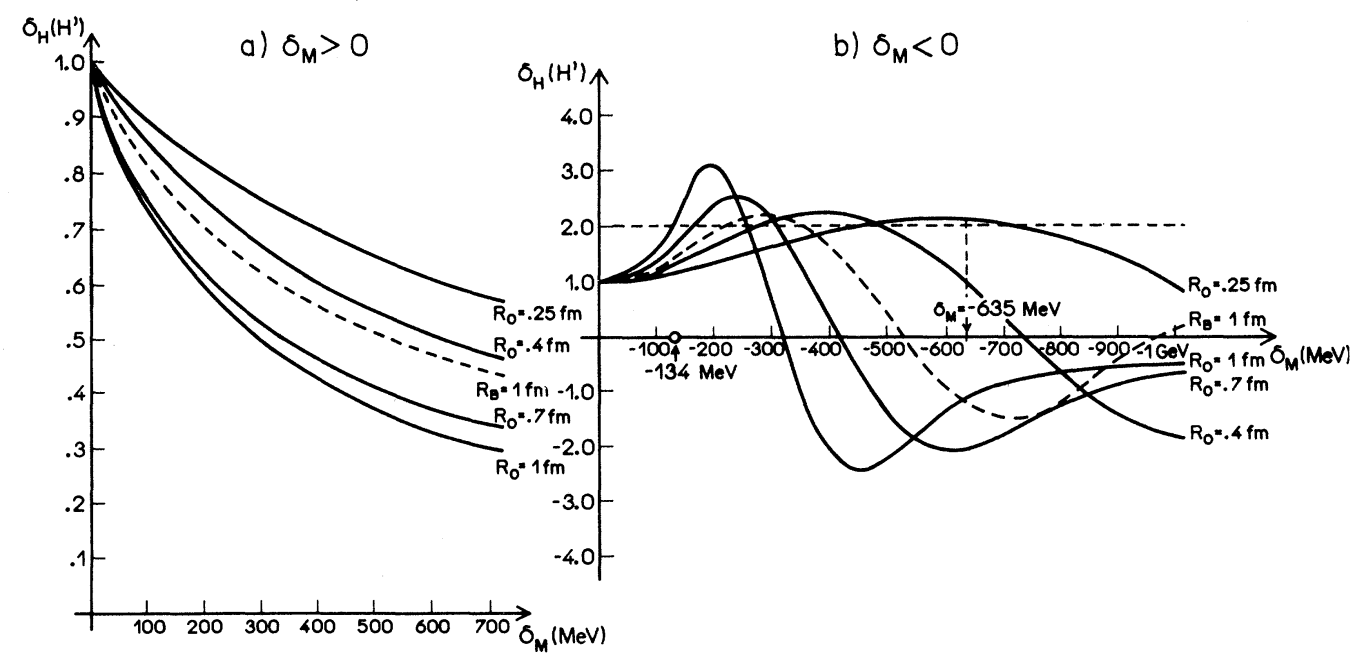

FIG. 2. Intermediate-mass correction $\delta_{H}\left(H^{\prime}\right)$ as a function of the mass difference $\delta_{M}=M_{H^{\prime}}-M_{H}$ for several values of $R_{0}$. The dashed curve refers to the bag model with $R_{b}=1 \mathrm{fm}$. 
given by Eq. (19b). In performing the numerical integrations in Eq. (18), the principal value was taken whenever a pole at $x=d^{2}-1$ appears.

It is interesting to note that in the case of the bag model, the parameter which controls the behavior of the curves is the bag radius $R_{b}$, while in our model is the parameter $R_{0}$, the "Gaussian radius" associated to the single quarks. Notice that when smaller values of $R_{0}$ are taken, the curves for $\delta_{M}<0$ stay in the upper region of Fig. 2(b), where $\delta_{H}\left(H^{\prime}\right)$ is positive. A similar behavior is found in the bag case. We have recently shown in a preliminary work ${ }^{12}$ that, in order to describe correctly the $\rho-\omega$ mass difference, both in sign and magnitude, it is necessary to take sufficiently small values for the radius of the mesons, as compared with those of the baryons. This point will be better clarified afterwards. Now, we shall present our results.

\section{THE IMMD'S: ASSUMPTIONS AND RESULTS}

Our results are based on Eq. (7), which allows us to calculate the physical hadron masses taking into account the several energy corrections effects, as given by Eqs. (8), (9), and (12), including self-energy terms, whose coefficients are given in Table $\mathbf{I}(\mathrm{a})$.

In a previous work, ${ }^{12}$ the effects of the intermediate states to the pion-energy corrections have also been incorporated. This was necessary for a correct description of the mass spectrum of the mesonic sector. Furthermore, it was shown that the $\rho$ - $\omega$ mass splitting can be obtained both in sign and magnitude only when the appropriate corrections due to the nondegenerate intermediate states are introduced. Besides, a parametrization of the mesonic sector different from that of the baryons was required. In order to make this point clearer, let us briefly recall here the main features of that analysis, because they will be of importance for the understanding of this work.

In the mechanism of the $\rho$ - $\omega$ mass difference, a decisive role is played by the diagram of the $\rho$ meson containing two pions in Fig. 1(b). It is well known that nonchiral models lead to $M_{\rho}=M_{\omega}$ (Ref. 3 ). On the other hand, a direct application of the pion corrections by means of Eq. (12) would lead to the result $M_{\rho}>M_{\omega}$ (Ref. 13), while experimentally we have $M_{\rho}<M_{\omega}$. This wrong result is a consequence of the use in Eq. (12) of the uncorrected spin-isospin matrix element $C(H)$, defined by Eq. (13). As can be seen from Table II, $C(\omega)=24>C(\rho)=16$ and then, as the pion-cloud energy corrections are subtractive, one finds $M_{\omega}<M_{\rho}$. By using the corrected matrix elements, defined by Eqs. (15) and (16), the situation changes. When $C(\rho)=16$ is broken to $C(\rho \omega)=8$ plus $C(\rho \pi)=8$ and the corrections $\delta_{\rho}\left(H^{\prime}\right)$ are applied, we get $\delta_{\rho}(\pi)>2.00$. Consequently, $C^{\prime}(\rho)>24$ and thus the right splitting, with the lightest $\rho$, is found.

It must be noted that this result can be achieved only if the radius $R_{0}$ for mesons is adjusted to a conveniently small value, corresponding to a curve passing in the upper region of Fig. 2(b), with $\delta_{H}\left(H^{\prime}\right)>2$ for $\delta_{M}=M_{H^{\prime}}-M_{H}=M_{\pi}-M_{\rho}=-635 \mathrm{MeV}$. We have fitted the $\rho-\omega$ mass splitting with $R_{0}=0.27 \mathrm{fm}$ while for the baryons $N$ and $\Delta$ we had found $R_{0}=0.58 \mathrm{fm}$. Then, a different parametrization for the mesonic sector came out.

Furthermore, we note that in Ref. 12 our fitting of the light baryons and mesons was made with the aid of the naive color relation ${ }^{5} V_{q q}=\frac{1}{2} V_{q \bar{q}}$, which encouraged us to try $\langle V(r)\rangle_{\text {baryon }} \cong \frac{1}{2}\langle V(r)\rangle_{\text {meson. }}$. We recall that, although the color relation appears inappropriate in the confinement region where multigluon processes dominate, there is some indication in the literature of its approximate validity ${ }^{14}$ in the present context.

After these preliminary considerations, let us concentrate our attention to the IMMD problem. We started the present fitting using a set of parameters with values close to those determined in the $\rho$ - $\omega$ mass splitting. The new feature is that now we have $m_{u} \neq m_{d}$. This induces contributions of strong and electromagnetic origin to the mass differences, as we already pointed out. The best fittings obtained for the hadron masses are given in Tables III(a) and III(b), where one can analyze the several energy corrections which contribute to the physical hadron masses, calculated according to Eq. (7). In Table IV, the corresponding IMMD's are presented and compared with the available experimental data. ${ }^{15}$

In our previous work, ${ }^{12}$ we had used $m_{u}=m_{d}=7 \mathrm{MeV}$. Now, by means of slight variations around this value, we determine $m_{u}$ and $m_{d}$ in order to fit the neutron and proton masses. We have found $m_{u}=5.78 \mathrm{MeV}$ and

TABLE II. Corrections to the matrix elements $C(H)$ due to the nondegenerate intermediate states, Eqs. (15) and (16), calculated in our previous work, Ref. 12.

\begin{tabular}{cccccccc}
\hline \hline $\boldsymbol{H}$ & $\boldsymbol{H}^{\prime}$ & $\boldsymbol{C}(\boldsymbol{H})$ & $C\left(\boldsymbol{H} \boldsymbol{H}^{\prime}\right)$ & $\delta_{M}=M_{H^{\prime}}-M_{H}$ & $\delta_{H}\left(H^{\prime}\right)$ & $\delta_{H}\left(H^{\prime}\right) C\left(H H^{\prime}\right)$ & $C^{\prime}(H)$ \\
\hline$\rho$ & $\omega$ & 16 & 8 & 13 & 0.97 & 7.76 & 24.32 \\
& $\pi$ & & 8 & -635 & 2.07 & 16.56 & \\
$\omega$ & $\rho$ & 24 & 24 & -13 & 1.00 & 24.00 & 24.00 \\
$\pi$ & $\rho$ & 24 & 24 & 635 & 0.572 & 13.73 & 13.73 \\
$N$ & $N$ & 57 & 25 & 0 & 1 & 25 & 44.26 \\
& $\Delta$ & & 32 & 294 & 0.602 & 19.26 & \\
$\Delta$ & $\Delta$ & 33 & 25 & 0 & 1 & 25 & 44.44 \\
& $N$ & & 8 & -294 & 2.43 & 19.44 & \\
\hline \hline
\end{tabular}


TABLE III. Energy corrections and hadron masses (in MeV). The Gaussian radius $R_{0}$ and the corresponding corrected matrix element $C^{\prime}(H)$ for each hadron are also given in the tables. In (a) the matrix elements $C^{\prime}(H)$ are those obtained in Ref. 12 (see also Table II), while those in (b) have been calculated with a nondegenerate triplet of Goldstone pions. The parameters are given below, in the tables. In both fittings we have $V_{0}$ (baryons) $=\frac{1}{2} V_{0}$ (mesons) $=71 \mathrm{MeV}$.

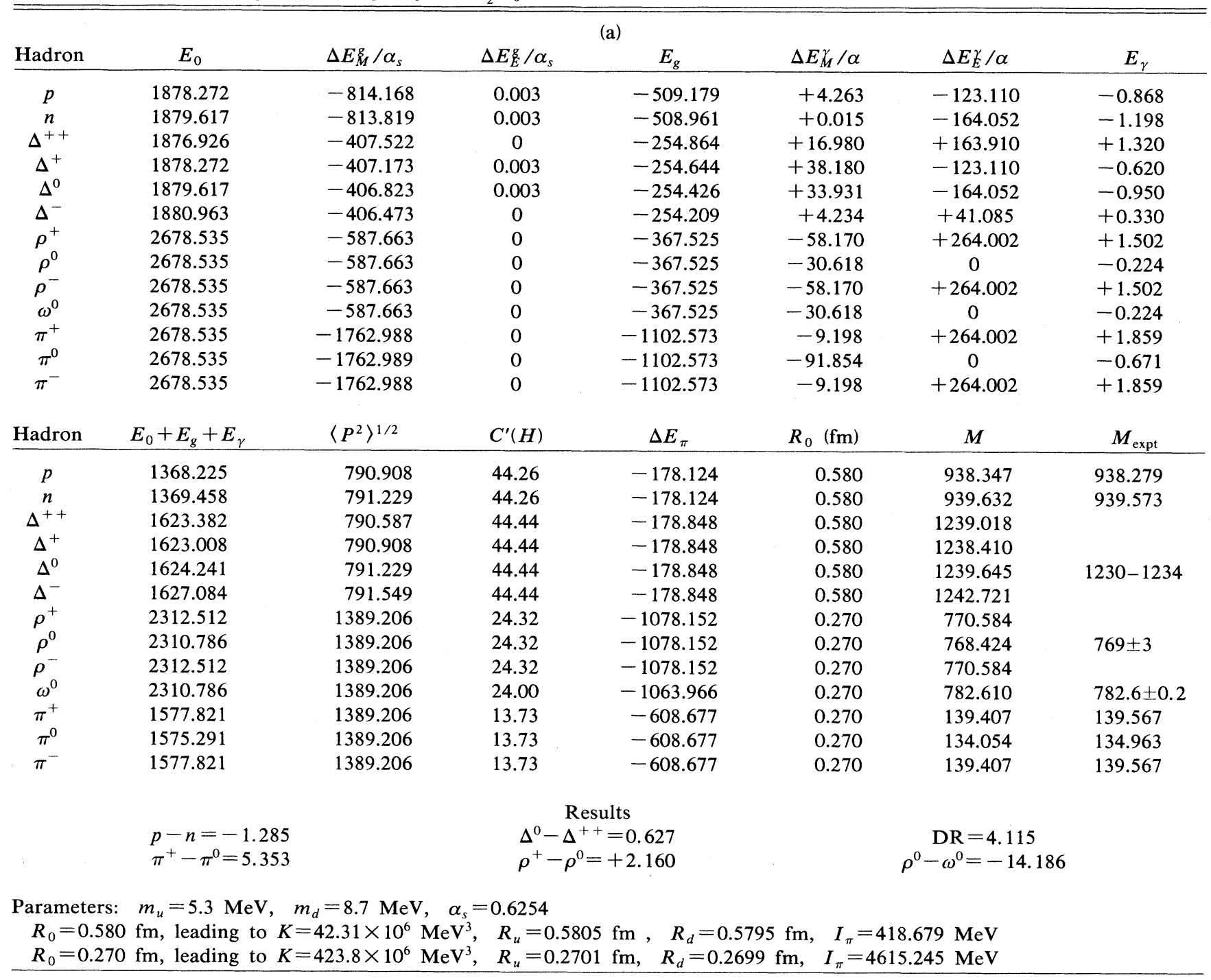

$m_{d}=9.50 \mathrm{MeV}$, leading to $\bar{m}=\frac{1}{2}\left(m_{u}+m_{d}\right)=7.64 \mathrm{MeV}$ with $m_{d}-m_{u}=3.72 \mathrm{MeV}$. These values are in good agreement with the predictions of the standard model, which gives $m_{u} \approx 5.5 \mathrm{MeV}, m_{d} \approx 10 \mathrm{MeV}$, and $m_{s} \approx 200$ $\mathrm{MeV}$ (Ref. 16). We note that this agreement with our results was not necessarily expected, even in the framework of a relativistic quark model. One sees that it was the introduction of the several energy corrections which naturally allowed us to obtain values for the quark masses close to the QCD expectations.

Although the parameters which enter in Table III(a) have been fixed in our previous work, ${ }^{12}$ this time we had to improve our precision in the calculations in order to describe the IMMD's accurately. As compared with our former works, a different feature is that now, instead of using the spring constant $K$, we parametrized our calculations in function of the Gaussian radius $R_{0}$. In our previous work we had found $R_{0}=0.58 \mathrm{fm}$ for the baryons $N$ and $\Delta$ and $R_{0}=0.27 \mathrm{fm}$ for the mesons $\rho, \omega$, and $\pi$. The present fitting was achieved through slight variations around these values. As can be seen from Table III, this time we have fitted the baryon $\Delta$ with a radius a little larger than the nucleon. We have $R_{0}^{N}=0.580 \mathrm{fm}$ and $R_{0}^{\Delta}=0.586 \mathrm{fm}$. Setting $R_{b}^{2}=\Sigma_{i} R_{i}^{2}$, we find $R_{b} \cong \sqrt{3} R_{0}$ for the corresponding bag radius, and then results $R_{b}^{N}=1.00 \mathrm{fm}$ and $R_{b}^{\Delta}=1.01 \mathrm{fm}$. In the framework of the bag model, Hwang ${ }^{7}$ has also taken into account $R_{b}$ (octet) $<R_{b}$ (decuplet), with $R_{b}^{N}=0.987 \mathrm{fm}$ and $R_{b}^{\Delta}$ $=1.081 \mathrm{fm}$.

We recall that already in the original MIT bag model ${ }^{8}$ 
TABLE III. (Continued).

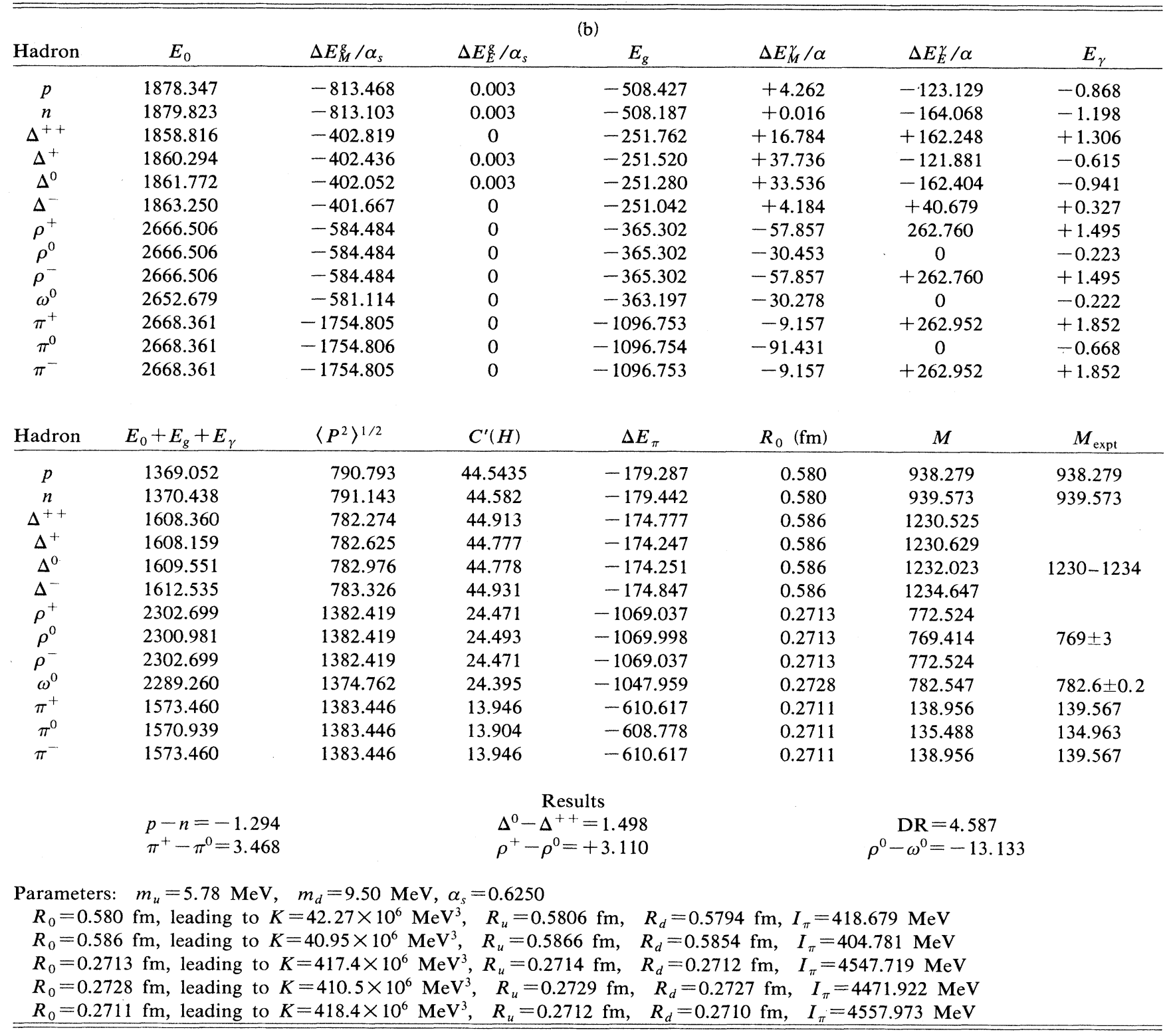

the radius of the bag $R_{b}$ for the nucleon and $\Delta$ resonance are different. In fact, one has $R_{\Delta}>R_{N}$, a result which is obtained from the nonlinear boundary condition, by minimization of the total bag energy when the magnetic contribution of one-gluon exchange is taken into account. In the present model, of course, no such boundary conditions exist. However, an analogous relation involving the Gaussian radius $R_{0}$ of an ordinary quark in the nucleon and $\Delta$ should also hold due to the repulsive effect of the spin-spin interactions, arising out of the one-gluon exchange, in the case of the $\Delta$ resonance. Effects of this kind, in the framework of a relativistic quark potential model with Gaussian wave functions have been discussed in detail by Itoh et al. ${ }^{9}$ as mentioned before.
A similar effect is found in the mesonic sector because vector mesons are also subject to spin-spin repulsive interactions. Consequently, one could expect $R(\omega, \rho)$ $>R(\pi)$. However, as the $\rho$-meson assumes an intermediate state as a virtual pion [plus another Goldstone-type pion; see Fig. 1(b)] the spin-spin repulsive effect appears somewhat suppressed. In fact, in our fitting we have $R_{0}^{\omega}=0.273 \mathrm{fm}>R_{0}^{\rho} \cong R_{0}^{\pi} \cong 0.271 \mathrm{fm}$, and we note that, apart from the above considerations and although $R_{0}^{\omega}$ is just slightly larger than $R_{0}^{\rho}$, these values were fitted in order to maintain the good fitting of the $\rho-\omega$ mass splitting obtained in our earlier work.

We also want to call attention that in the present work the corrected spin-isospin matrix element $C^{\prime}(H)$ were re- 
TABLE IV. Several results obtained for the light hadrons IMMD's. DR is the "delta relation," defined in the text. The last line corresponds to the fit of Table IIIb, where we introduced the nondegenerate triplet of Goldstone pions.

\begin{tabular}{|c|c|c|c|c|c|c|c|c|}
\hline$m_{u}$ & $m_{d}$ & $\bar{m}$ & $m_{d}-m_{u}$ & $p-n$ & $\pi^{+}-\pi^{0}$ & $\Delta^{0}-\Delta^{++}$ & DR & $\rho^{+}-\rho^{0}$ \\
\hline 5.0 & 8.2 & 6.6 & 3.2 & -1.183 & 5.349 & 0.432 & 3.790 & 2.159 \\
\hline 5.3 & 8.7 & 7.0 & 3.4 & -1.285 & 5.345 & 0.626 & 4.113 & 2.160 \\
\hline 6.0 & 9.4 & 7.7 & 3.4 & -1.290 & 5.337 & 0.635 & 4.129 & 2.159 \\
\hline 6.3 & 9.7 & 8.0 & 3.4 & -1.292 & 5.334 & 0.639 & 4.136 & 2.160 \\
\hline 6.6 & 10.0 & 8.3 & 3.4 & -1.294 & 5.330 & 0.643 & 4.142 & 2.159 \\
\hline 7.0 & 10.4 & 8.7 & 3.4 & -1.296 & 5.325 & 0.647 & 4.150 & 2.160 \\
\hline 5.2 & 8.8 & 7.0 & 3.6 & -1.384 & 5.345 & 0.816 & 4.430 & 2.160 \\
\hline 5.78 & 9.50 & 7.64 & 3.72 & -1.294 & 3.468 & 1.498 & 4.587 & 3.110 \\
\hline \multicolumn{4}{|c|}{ Experimental value (Ref. 15) } & -1.29332 & 4.604 & $2.7 \pm 0.3$ & $4.6 \pm 0.2$ & $-0.3 \pm 2.2$ \\
\hline
\end{tabular}

calculated with higher precision, giving rise to the values in Table III(b). The main improvement was the introduction of a triplet of Goldstone pions in our formulation of the pion-cloud corrections. This is to be understood in the following sense: In our previous work, ${ }^{12}$ as well as in former formulations of the pion-energy corrections, ${ }^{4,11}$ the mass of the Goldstone-pion was introduced into the calculations with one fixed value; for instance, $m_{\pi}=134$ $\mathrm{MeV}$ in Ref. 12. However, if we consider a breaking of the diagrams in Fig. 1 into their available charged states, we have the presence of neutral and charged Goldstone pions, with masses $m_{\pi^{0}}=134 \mathrm{MeV}$ and $m_{\pi^{ \pm}}=139 \mathrm{MeV}$, respectively. The introduction of the charged pions with $m_{\pi}=139 \mathrm{MeV}$ modifies significatively the evaluation of $I_{\pi}[\mathrm{Eq} .(14)]$ and the intermediate corrections $\delta_{H}\left(H^{\prime}\right)$ [Eq. (17)].

Now, to conclude this section, we would like to discuss in some detail the results obtained for the IMMD's.

\section{A. Nucleon $[p(u u d)-n(u d d)]$}

As can be seen from Tables III and IV, the protonneutron mass difference $(p-n)$ is almost completely originated from the kinetic part of the energy, arising out of the quark mass difference $\left(m_{u}-m_{d}\right)$. In Table III one sees that

$$
\begin{aligned}
& E_{0}(n)>E_{0}(p), \quad E_{0}(n)-E_{0}(p) \cong 1.5 \mathrm{MeV} \\
& \left\langle P^{2}\right\rangle_{n}^{1 / 2}>\left\langle P^{2}\right\rangle_{p}^{1 / 2}, \quad\left\langle P^{2}\right\rangle_{n}^{1 / 2}-\left\langle P^{2}\right\rangle_{p}^{1 / 2} \cong 0.3 \mathrm{MeV}
\end{aligned}
$$

in such a way that from the kinetic part will result approximately $M_{n}-M_{p} \cong 1.2 \mathrm{MeV}$. The energy corrections due to gluons and photons are small and cancel each other in the mass difference. The gluon correction is slightly higher in the proton while the photon correction is slightly higher in the neutron, namely,

$$
\begin{aligned}
& \left|E_{g}(p)\right|>\left|E_{g}(n)\right|, \quad E_{g}(p)-E_{g}(n) \cong-0.3 \mathrm{MeV}, \\
& \left|E_{\gamma}(n)\right|>\left|E_{\gamma}(p)\right|, \quad E_{\gamma}(p)-E_{\gamma}(n) \cong+0.3 \mathrm{MeV},
\end{aligned}
$$

so that $\left(E_{g}+E_{\gamma}\right)_{p}-\left(E_{g}+E_{\gamma}\right)_{n} \cong 0$. In addition, the pion-cloud effect is similar in the proton and neutron, $\Delta E_{\pi}(p) \cong \Delta E_{\pi}(n)$, and does not contribute significatively to the mass difference $n-p$.

Thus, one sees that $p-n=-1.293 \mathrm{MeV} \cong \frac{1}{3}\left(m_{u}\right.$ $-m_{d}$ ), with $m_{d}-m_{u} \cong 3.4$ to $3.8 \mathrm{MeV}=3.6 \pm 0.2 \mathrm{MeV}$ in the present fittings, leading to the result

$$
n-p \cong \frac{1}{3}\left(m_{d}-m_{u}\right) \cong 1.2 \mathrm{MeV}
$$

\section{B. Baryons $\Delta$}

The understanding of the $\Delta$ is more complicated than the nucleon because in the $\Delta$ 's there is a mixing of the several energy corrections. In particular, the effect of the pion-cloud (which is very sensitive to the adjusted radius $R_{0}$ ) can even alter the ordering of the masses. Generally, for baryons the most positively charged states are lighter because $m_{u}<m_{d}$. However, depending on the fitted parameters, the pionic energy corrections can make, for instance, the $\Delta^{+}(d u u)$ lighter than the $\Delta^{++}(u u u)$ (see Table III).

Experimentally, a complete determination of the mass differences among the charged $\Delta$ states is not available. However, it is known that

$$
\begin{aligned}
& \Delta^{0}-\Delta^{++}=2.7 \pm 0.3 \mathrm{MeV} \quad(\text { Refs. } 15 \text { and } 17) \\
& \mathrm{DR} \equiv\left(\Delta^{-}-\Delta^{++}\right)+\frac{1}{3}\left(\Delta^{0}-\Delta^{+}\right) \\
& \quad=4.6 \pm 0.2 \mathrm{MeV} \quad(\text { Ref. } 18) .
\end{aligned}
$$

The first of these relations, $\Delta^{0}-\Delta^{++}$, is harder to describe. The pionic correction seems to participate with at least $0.5 \mathrm{MeV}$ in this mass difference. In Table $\mathrm{V}$, we compare the results obtained by different calculations. One sees that our results have been a little more improved because of the introduction of the pion-cloud with the effect of the intermediate states. We believe that a more precise description of the $\Delta^{0}-\Delta^{++}$mass difference would require slightly different radii among them, an effect possibly originated from Coulomb forces, which 
TABLE V. Results obtained for the $\Delta$ 's by means of different calculations, in comparison with the experimental data.

\begin{tabular}{|c|c|c|c|}
\hline References & Kind of treatment & $\Delta^{0}-\Delta^{++}$ & DR \\
\hline $\begin{array}{l}\text { Bickerstaff and } \\
\text { Thomas, Ref. } 19\end{array}$ & $\begin{array}{l}\text { Without pion-cloud } \\
\text { corrections }\end{array}$ & 1.15 & 4.47 \\
\hline $\begin{array}{l}\text { C. Itoh et al., } \\
\text { Ref. } 19\end{array}$ & $\begin{array}{l}\text { Without pion-cloud } \\
\text { corrections }\end{array}$ & 0.85 & 4.95 \\
\hline $\begin{array}{l}\text { W.Y.P. Hwang, } \\
\text { Ref. } 7\end{array}$ & $\begin{array}{l}\text { Pion-cloud corrections } \\
\text { with degenerate } \\
\text { intermediate states }\end{array}$ & 0.0 & 4.2 \\
\hline Present work & $\begin{array}{l}\text { Pion-cloud corrections } \\
\text { including nondegenerate } \\
\text { intermediate states } \\
\text { [fitting of Table III(a)] }\end{array}$ & 0.627 & 4.115 \\
\hline Present work & $\begin{array}{l}\text { Pion-cloud corrections } \\
\text { including nondegenerate } \\
\text { intermediate states, } \\
\text { calculated with a non- } \\
\text { degenerate triplet of } \\
\text { Goldstone pions [fitting } \\
\text { of Table III(b)] }\end{array}$ & 1.498 & 4.587 \\
\hline Experimental value & & $\begin{array}{l}2.7 \pm 0.3 \text { (Ref. 15) } \\
2.4 \pm 0.5 \text { (Ref. 17) }\end{array}$ & $4.6 \pm 0.2($ Ref. 18$)$ \\
\hline
\end{tabular}

tend to deform differently the $\Delta^{0}$ and the $\Delta^{++}$charged states.

The second relation, which we call here "delta relation" (DR), is due to Pedroni et al. ${ }^{18}$ and is more easily described. Once fitted the Gaussian radius $R_{0}$ and the quark masses, one sees that this is a very stable relation around the value $4.5 \pm 0.4 \mathrm{MeV}$, as can be seen from Tables IV and V. It seems that, apart of the uncertainties inherent in any description, the several effects compensate each other giving rise to a reasonable and stable result for it.

\section{Pions}

Pion masses are known with good precision, the charged states being heavier than the neutral one. It is clear that this mass difference arises fundamentally from an electromagnetic effect. However, small contributions from pion-cloud correction exist if the Goldstone boson is treated as a non-degenerate triplet. In fact, from Tables III(a) and III(b) one sees that

$E_{0}\left(\pi^{+}\right)=E_{0}\left(\pi^{0}\right), \quad\left\langle P^{2}\right\rangle_{\pi^{+}}=\left\langle P^{2}\right\rangle_{\pi^{0}}, \quad E_{g}\left(\pi^{+}\right)=E_{g}\left(\pi^{0}\right)$

while $E_{\gamma}\left(\pi^{0}\right) \cong-0.7 \mathrm{MeV}<E_{\gamma}\left(\pi^{+}\right) \cong 1.8 \mathrm{MeV}$ leading to a lighter $\pi^{0}$.

Notice that in our first fitting [see Table III(a)], with $\Delta E_{\pi}\left(\pi^{+}\right)=\Delta E_{\pi}\left(\pi^{0}\right)$ we obtained $\pi^{+}-\pi^{0} \cong 5.3 \mathrm{MeV}$, a result only a little larger than the experimental value. By including the nondegenerate Goldstone pions [Table III(b)] we found $C^{\prime}\left(\pi^{+}\right)>C^{\prime}\left(\pi^{0}\right)$ leading to
$\Delta E_{\pi}\left(\pi^{+}\right)>\Delta E_{\pi}\left(\pi^{0}\right)$. As a result, the mass difference decreased to $\pi^{+}-\pi^{0} \cong 3.5 \mathrm{MeV}$. We note that although the incorporation of this effect was performed in an approximate way, it is gratifying that it leads to results in the right direction. Perhaps Coulomb-breaking effects, similar to those discussed in Ref. 9, are also important in this case, because the fitting of the radius $R_{0}$ is very sensitive in this calculation, mainly affecting the calculated values of the matrix elements $C^{\prime}\left(\pi^{+}\right)$and $C^{\prime}\left(\pi^{0}\right)$, whose adjustment with the right intensity should yield the experimental value $\pi^{+}-\pi^{0}=4.604 \mathrm{MeV}$.

\section{Mesons $\rho$}

Data on the IMMD's of the vector $\rho$ meson are much less precise. Actually, it is known that $m_{\rho^{0}}=769 \pm 3$ $\mathrm{MeV}$, with an indication that $\rho^{+}-\rho^{0}=-0.3 \pm 2.2 \mathrm{MeV}$ (Ref. 15).

We note that for nonstrange mesons the charged states are systematically heavier than the neutral states. This is due to the electromagnetic contribution. However, for the $\rho$-mesons the intermediate states take place and the pion-correction effect can diminish or even invert the sign of the mass difference $\rho^{+}-\rho^{0}$, in which case, $\rho^{0}$ would be heavier.

It is interesting to note that such an effect can only be described when the pion-cloud correction is introduced with the inclusion of a nondegenerate triplet of Goldstone pions, because, depending on the fitting, it can give $C^{\prime}\left(\rho^{0}\right)>C^{\prime}\left(\rho^{+}\right)$leading to a lighter $\rho^{0}$, or $C^{\prime}\left(\rho^{+}\right)$ $>C^{\prime}\left(\rho^{0}\right)$ leading to a lighter $\rho^{ \pm}$. 
In the present fitting we have $\rho^{0}$ lighter than the $\rho^{ \pm}$. However, we remark that this is not a conclusive result. A more detailed treatment of the intermediate states may well give rise to $m_{\rho^{0}}>m_{\rho^{ \pm}}$.

\section{CONCLUSIONS}

As we have seen, the $m_{d}-m_{u}$ mass difference plays an essential role in the calculation of the IMMD's. The incorporation in the present model of center-of-mass, onegluon-exchange, photon-exchange, and pion-cloud corrections allowed us to adjust the quark masses with values close to the QCD expectations. We have also found that using $m_{d}-m_{u} \approx 3.6 \mathrm{MeV}$ the $n-p$ mass difference can be properly fitted. While in this case the main contribution for the mass difference is originated from the kinetic part of the energy, in the case of $\pi^{+}-\pi^{0}$ the main effect is due to the electromagnetic contributions. As far as the $\Delta$ 's and $\rho$ 's are concerned, other contributions also participate and pion-cloud effects take place. The intermediate states play a fundamental role in this analysis, showing its particular importance in the description of the $\rho^{0}-\omega^{0}$ and $\rho^{0}-\rho^{+}$mass differences. The sensitivity of the fitting can be increased by means of the introduction of more subtle effects, such as deformations of the quark "core" due to Coulomb forces or, otherwise, the introduction of a triplet of Goldstone pions, as we have discussed here.

We would like to recall that, in chiral models, as ours, the pions assume a dual role. When chiral symmetry is introduced, they are viewed as elementary Goldstone particles which assume masses $m_{\pi^{0}}=134 \mathrm{MeV}$ and $m_{\pi^{+}}=139 \mathrm{MeV}$ in the PCAC (partial conservation of axial-vector current) limit. On the other hand, they can be considered as a quark-antiquark bound state belonging to the pseudoscalar flavor nonet. Thus, as ordinary mesons, their masses can be calculated in the model. It is a gratifying result that, even in a simple model as ours, the $q \bar{q}$ pion appears consistently with a mass $M_{\pi}(q \bar{q})$ very nearly equal to the mass values $m_{\pi}$ taken for the Goldstone pions.

It is interesting to note that, for a successful pion-mass calculation, it is fundamental to take an adequate Gaussian radius. As for the $\rho$ and $\omega$ mesons, the Gaussian radius for the constituent quarks in the pion were also $R_{0}=0.27 \mathrm{fm}$. Consequently, using a simple quadratic relation $R_{b}^{2} \cong \sum_{i} R_{i}^{2}$, the pion-bag radius would result $R_{b} \cong \sqrt{2} R_{0}=0.38 \mathrm{fm}$. Curiously, this value is in agreement with other references, ${ }^{20}$ although obtained in completely different frameworks. Bernard and Meissner ${ }^{20}$ have analyzed the electromagnetic structure of the pion and the kaon and found an "intrinsic pion radius" of 0.37 $\mathrm{fm}$. In another approach, Brown, Rho, and Weise ${ }^{20}$ described the pion form factor with the contribution of the pion core parametrized by an intrinsic radius of $0.35 \mathrm{fm}$. These values seem to corroborate our fitting of the lightmeson sector.

We note that we have not considered the $\eta$ and $\eta^{\prime}$ pseudoscalar-meson masses. The reason is that, in view of the limited scope of this work, annihilation forces, which play an important role for those mesons, were not discussed. However, we remark that both $\eta$ and $\eta^{\prime}$ are devoid of pion clouds. This statement is a consequence of the fact that, independently of the mixing angle, all the matrix elements $C\left(H H^{\prime}\right)$ with $H=\eta$ or $\eta^{\prime}$ vanish, as can be easily checked by direct calculation. This fact tends to increase the $\eta$ and $\eta^{\prime}$ masses, in agreement with experience.

Finally, a few words should be addressed to the IMMD's of the kaons. The experimental results ${ }^{15}$ show that the neutral states are heavier than the charged ones: namely, $K^{0}-K^{+}=4.05 \pm 0.07 \mathrm{MeV}$ and $K^{0 *}-K^{+*}$ $=4.4 \pm 0.5 \mathrm{MeV}$. However, both photon and pionexchange energy corrections lead to contributions in the opposite direction. We note that to explain the kaons' IMMD's it is necessary to take sufficiently large values for their radii, in order to increase the OGE and kinetic energy contributions. In fact, for a radius $R_{0}>0.35 \mathrm{fm}$ $\left(R_{b}>0.45 \mathrm{fm}\right)$ one can obtain the kaon mass differences with the correct sign, but not their magnitudes (the best result we have obtained is $K^{0}-K^{+}=+0.623 \mathrm{MeV}$ and $K^{0 *}-K^{+*}=+0.473 \mathrm{MeV}$, for $R_{0} \cong 0.56 \mathrm{fm}$ ). We note that there is an independent "phenomenological" indication that when strange quarks are present, the bag radius $R_{b}$ is frozen at a value substantially larger than $0.5 \mathrm{fm}$ (Ref. 21). This effect, together with other additional effects, such as the quark-core deformations due to Coulomb forces [which act differently in the $K^{0}(d \bar{s})$ and $K^{+}(u \bar{s})$ mesons] might explain the kaons' IMMD's.

Furthermore, different interesting effects may take place when the IMMD's of heavy hadron systems are considered. For instance, one has to explain the $\Sigma_{c}^{++}-\Sigma_{c}^{0}$ mass difference, for which different results have been obtained. ${ }^{22}$ As we have shown, pion-cloud effects including contributions of intermediate states are instrumental to describe the light hadron IMMD's. Likewise, those corrections may also play a decisive role to the understanding of IMMD's of $Q q q, Q Q q$ baryons, and $Q \bar{q}$-meson systems, due to the coupling of the pion-field with the light quarks $q$. An extension of this work treating the IMMD's of the heavy hadron sector is scheduled for a later date.

\section{ACKNOWLEDGMENTS}

One of us (B.E.P.) is grateful to Fundação de Amparo e Pesquisa do Estado de Săo Paulo for financial support.
${ }^{1}$ For a first reference on the role of the mass difference between down and up quarks in strong interactions, see Nathan Isgur, Phys. Rev. D 21, 779 (1980).

${ }^{2}$ P. Leal Ferreira, J. A. Helayel, and N. Zagury, Nuovo Cimento
A 55, 215 (1980); R. Tegen, R. Brockmann, and W. Weise, Z. Phys. A 307, 339 (1982).

${ }^{3}$ N. Barik, B. K. Dash, and M. Das, Phys. Rev. D 31, 1652 (1985); B. E. Palladino and P. Leal Ferreira, ibid. 34, 2168 
(1986).

${ }^{4}$ N. Barik and B. K. Dash, Phys. Rev. D 33, 1925 (1986); B. E. Palladino and P. Leal Ferreira, Rev. Bras. Fis. 18, 478 (1988).

${ }^{5}$ See, e.g., D. Flamm and F. Schöberl, Quark Model of Elementary Particles (Gordon and Breach, New York, 1982), Vol. 1.

${ }^{6}$ S. Theberge, A. W. Thomas, and G. A. Miller, Phys. Rev. D 22, 2838 (1980); A. W. Thomas, S. Theberge, and G. A. Miller, ibid. 24, 216 (1981); L. R. Dodd, A. W. Thomas, and R. F. Alvarez-Estrada, ibid. 24, 1961 (1981).

${ }^{7}$ W. Y. P. Hwang, Phys. Rev. D 31, 2826 (1985).

${ }^{8}$ T. De Grand, R. L. Jaffe, K. Johnson, and J. Kiskis, Phys. Rev. D 12, 2060 (1975).

${ }^{9}$ C. Itoh, T. Minamikawa, K. Miura, and T. Watanabe, Prog. Theor. Phys. 61, 548 (1979).

${ }^{10}$ M. G. do Amaral and N. Zagury, Phys. Rev. D 26, 3119 (1982).

${ }^{11}$ F. Myhrer, G. E. Brown, and Z. Xu, Nucl. Phys. A362, 317 (1981).

${ }^{12}$ B. E. Palladino and P. Leal Ferreira, Rev. Bras. Fis. (to be published).

${ }^{13}$ N. Barik, B. K. Dash, and P. C. Dash, Pramãna J. Phys. 29, 543 (1987).

${ }^{14}$ H. G. Dosh and V. F. Müller, Nucl. Phys. B116, 470 (1976); see also R. Sommer and J. Wosiek, Phys. Lett. 149B, 197
(1984).

${ }^{15}$ Particle Data Group, M. Aguilar-Benitez et al., Phys. Lett. 170B, 1 (1986).

${ }^{16}$ These values have been estimated from the work of J. Gasser and H. Leutwyler, Phys. Rep. 87, 77 (1982), and references therein; see also S. Narison, Revista del Nuovo Cimento 10, 1 (1987).

${ }^{17}$ S. Capstick, Phys. Rev. D 36, 2800 (1987).

${ }^{18}$ E. Pedroni et al., Nucl. Phys. A300, 321 (1978).

${ }^{19}$ R. P. Bickerstaff and A. W. Thomas, Phys. Rev. D 25, 1869 (1982); C. Itoh, T. Minamikawa, K. Miura, and T. Watanabe, Prog. Theor. Phys. 80, 208 (1988).

${ }^{20}$ V. Bernard and U. G. Meissner, Phys. Rev. Lett. 61, 2296 (1988); G. E. Brown, M. Rho, and W. Weise, Nucl. Phys. A454, 669 (1986).

${ }^{21}$ G. E. Brown et al., Z. Phys. A 331, 139 (1988); see also G. E. Brown and M. Rho, Comments Nucl. Part. Phys. 18, 1 (1988).

${ }^{22}$ W. Y. P. Hwang and D. B. Lichtenberg, Phys. Rev. D 35, 3526 (1987), and references therein; see also C. Itoh, T. Minamikawa, K. Miura, and T. Watanabe, Prog. Theor. Phys. 80, 208 (1988); S. N. Sinha, S. M. Sinha, M. Rahman, and D. Y. Kim, Phys. Lett. B 218, 333 (1989), and references therein. 